\section{Tratamiento de una maloclusión clase III en dentición decidua y mixta con expansión rápida palatina y máscara facial}

Treatment of class III malocclusion in primary and mixed dentition with rapid palatal expansion and facemask

\begin{abstract}
Resumen
Reportamos el seguimiento de un caso clínico de mordida cruzada que en un inicio comprometió el uso de un aparato de expansión palatina adhesivo de acrílico, que después de la estabilización del tratamiento y coincidente con el recambio dentario anterosuperior, sufrió recidiva de la mordida cruzada anterior, la cual fue corregida con el uso de máscara facial. El propósito de corregir la mordida cruzada en edades tempranas permite que se produzca mejoras en el patrón esquelético de clase III y reduce la cantidad de compensaciones dentales y discrepancias esqueléticas que generalmente están asociadas a la maloclusión esquelética severa en la adolescencia, sin embargo, la desventaja es el tiempo de tratamiento prolongado y la potencial inestabilidad de los cambios obtenidos. Independientemente de cuál sea el factor determinante de la recidiva, se recomienda la sobrecorreción del resalte y de la relación molar como una herramienta para la estabilidad a largo plazo.

Palabras clave: aparato de expansión palatina adhesivo de acrílico, máscara facial, mordida cruzada.
\end{abstract}

\begin{abstract}
We report the follow-up of a cross-bite clinical case which initially undertook the use of a Rigid acrylic-bonded palatal appliance that after stabilization treatment and coincident with the anterior tooth replacement, suffered a recurrence of anterior crossbite wich was corrected with the use of facemask. The purpose of correcting the crossbite at early ages allowed to drive improvements in the Class III skeletal pattern and reduces the amount of compensation to dental and skeletal discrepancies are usually associated with severe skeletal malocclusion in adolescence, however, the disadvantage is that the prolonged time treatment and the potential instability of the changes obtained. Independently the determinant of recurrence, is recommended the overcorrection overjet and molar relationship as a tool for long term stability.
\end{abstract}

Key words: rigid acrylic-bonded palatal appliance, facemask, crossbite.

\section{Introducción}

Una maloclusión de clase III durante el crecimiento constituye uno de los retos más difíciles con los que se puede encontrar el ortodoncista. Si no se tratan pueden empeorar, acabando en la mayoría de casos con intervenciones de cirugía ortognática una vez se llegue a la edad adulta.

Por esta razón, se recomienda el tratamiento ortodóncico interceptivo para reducir el porcentaje de casos que requerirán cirugía. Sin embargo, cuando se inicia este tipo de tratamiento a una edad temprana, a menudo hay un lapso significativo entre el final de la terapia con la máscara facial y el inicio de la ortodoncia definitiva.
Una maloclusión de clase III en desarrollo puede presentarse con una retrusión esquelética maxilar, una protrusión esquelética mandibular, o una combinación de ambas formas. ${ }^{1,2}$

La maloclusión clase III, afecta en alto grado la estética dentofacial y por tanto la autoestima del niño que se siente rechazado, condición que puede arrastrar hasta la edad adulta. Ello hace que los padres soliciten atención temprana al odontólogo familiar pero que debe ser remitido al especialista tan pronto sea detectado.

El éxito del tratamiento depende de cuatro áreas: la relación del maxilar y la mandíbula, la relación de los maxilares con el cráneo, la dimensión vertical y la edad del paciente. ${ }^{3}$
Casos Clínicos

\section{Luciano Carlos Soldevilla Galarza ${ }^{1}$, Arón Aliaga Del Castillo'}

${ }^{1}$ Dpto. Académico de Estomatología Pediátrica. Facultad de Odontología. Universidad Nacional Mayor de San Marcos.

\section{Correspondencia:}

Esp. Luciano Carlos Soldevilla Galarza. Facultad de Odontología. UNMSM. Av. Germán Amézaga s/n, Lima 1. Perú

Teléf.: 997-011-360

E-mail: lucianosol@yahoo.com
Fecha de recepción: 6-09-10

Fecha de aceptación: 5-04-11
Desde hace ańos, la tendencia ha sido el uso de máscaras faciales para protraer el maxilar en niños con maloclusión clase III. El desarrollo del sistema de suturas alrededor del complejo nasomaxilar implica cambios esqueléticos que se pueden obtener a una temprana edad, cuando aún la actividad de crecimiento a nivel de suturas en la región pterigoidea sigue siendo elevada. ${ }^{4} \mathrm{El}$ tratamiento con máscara facial comprende tres fases de tratamiento: expansión, protracción y retención. Una vez conseguidos los objetivos, los resultados deben ser mantenidos y evaluados en el tiempo para evitar recidivas o controlarlas según sea el caso. ${ }^{3}$

Los estudios sugieren que la recidiva ocurre en mayor o menor grado, y que 
además es inversamente proporcional a la duración de la estabilización, los cambios esqueléticos se presentan más estables que los dentales a razón de $4: 1 .^{5}$ Para algunos autores la recidiva se debe principalmente al crecimiento mandibular, ${ }^{6}$ para otros se debe a la deficiencia del crecimiento maxilar. ${ }^{7}$ Independientemente de cuál sea el factor determinante los autores recomiendan la sobrecorrección del resalte y de la relación molar como una herramienta para la estabilidad a largo plazo, ya que se ha comprobado que la máscara facial no normaliza el crecimiento. ${ }^{3}$

Cuando se estudia el efecto del tratamiento en maloclusiones clase III, es importante evaluar los resultados tanto a corto como a largo plazo, y establecer cuáles son los factores que juegan un rol importante en el mantenimiento de los mismos.

El objetivo de este artículo es remarcar la importancia del mantenimiento de los resultados obtenidos en el tratamiento de una maloclusión clase III, evaluándolos a corto, mediano y largo plazo.

\section{Reporte del caso}

Paciente masculino de 8 años 5 meses de edad, se presenta a consulta a la Clínica de la Facultad de Odontología de la Universidad Nacional Mayor de San Marcos, refiriendo la madre como motivo de consulta que su nińo presenta "una reaparición de mala mordida", el paciente es originario de la ciudad de Lima - Perú. Como antecedentes heredofamiliares refiere que varios miembros de su familia tienen el mismo problema. Como antecedente clínico refiere mordida cruzada anterior y posterior bilateral tratada con aparatología removible adhesiva a los 3 ańos 8 meses, en dentición decidua (Fig. 1-4). No presenta antecedentes personales patológicos. Tiene su esquema de vacunación completo y presenta alteraciones funcionales en la respiración y en la postura mandibular.

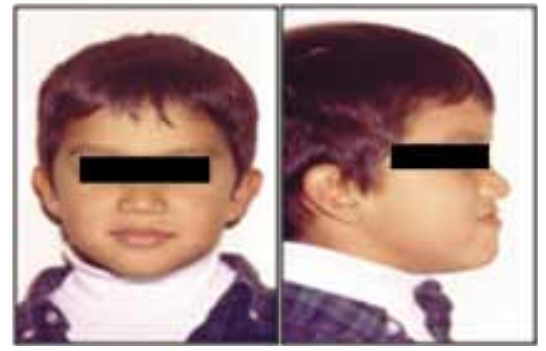

Fig. 1. Nińo de 3 ańos y 8 meses antes del tratamiento inicial.

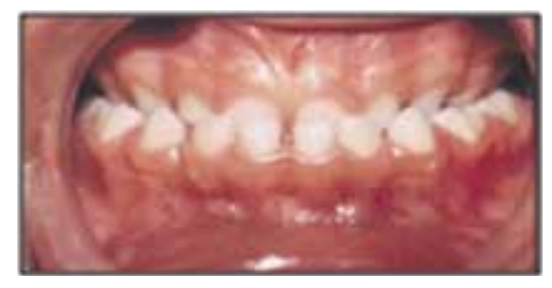

Fig. 2. Mordida cruzada anterior inicial.

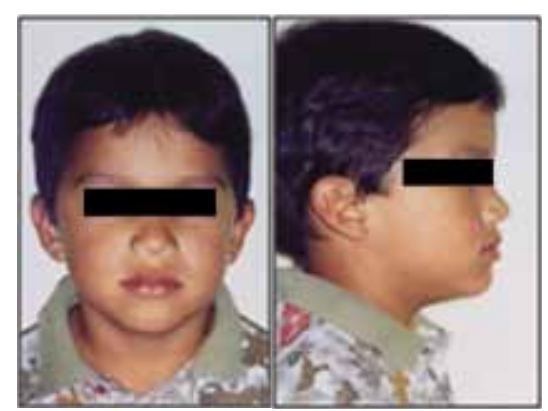

Fig. 3. Extraoral después del cuatro meses de tratamiento inicial en dentición temporal.

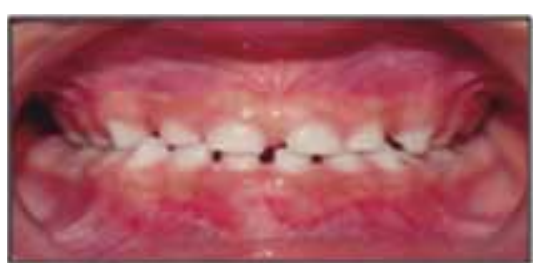

Fig. 4. Después del tratamiento inicial.

Se realiza un examen extraoral en el que se observa una implantación adecuada del cuero cabelludo, así como del pabellón auricular, el paciente presenta un perfil facial total y del tercio inferior cóncavo (Fig. 5).



Fig. 5. A los 8 años y 5 meses de edad.

Dentro de la exploración intraoral se evalúa el estado de los tejidos blandos y la salud periodontal sin que se observen datos clínicos patológicos, se realiza la valoración de los órganos dentarios presentando restauraciones con resina en las molares deciduas. Presenta arcos dentarios superior e inferior en dentición mixta (Fig. 6).

En la relación oclusal se observa una mordida cruzada anterior en máxima intercuspidación y desviación de la línea media en arcada inferior hacia la derecha de $0.5 \mathrm{~mm}$ (Fig. 7), una rela-

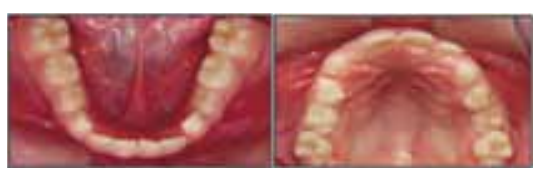

Fig. 6 .Aspecto oclusal intraoral.

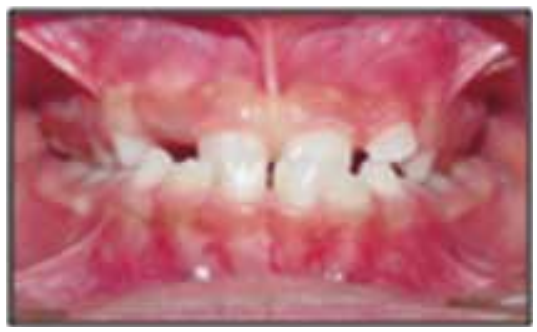

Fig. 7. Mordida cruzada anterior.

ción molar clase III derecha e izquierda. (Fig 8 y 9). En posición de relación céntrica no se consigue una relación anterior de borde a borde.

Marcada lingualización de los incisivos inferiores.

El objetivo principal del tratamiento fue brindar al paciente una atención ortodóncica interceptiva inmediata, que permitiera la corrección de la mordida cruzada anterior para devolver la funcionalidad de la oclusión que había adquirido hace 5 años. Dentro de esta fase ortodóncica los objetivos fueron: corregir la mordida cruzada anterior, mejorar el perfil facial, y corregir las alteraciones funcionales.

En el plan del tratamiento inicial se realizó la valoración ortodóncica: protracción maxilar con aparatología extraoral removible para corregir la mordida cruzada anterior (Fig. 10 y 11). Valoración funcional: Tratamiento otorrinolaringológico y fonoaudiológico.

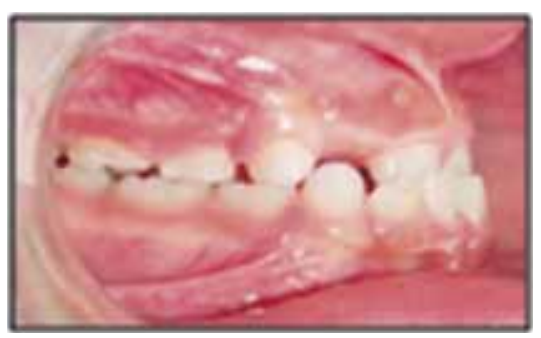

Fig. 8. Relación molar derecha en Clase III.

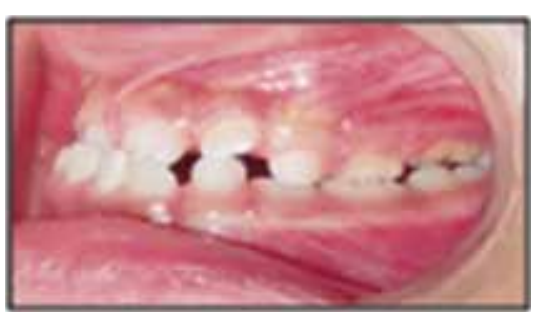

Fig. 9. Relación molar izquierda en Clase III. 


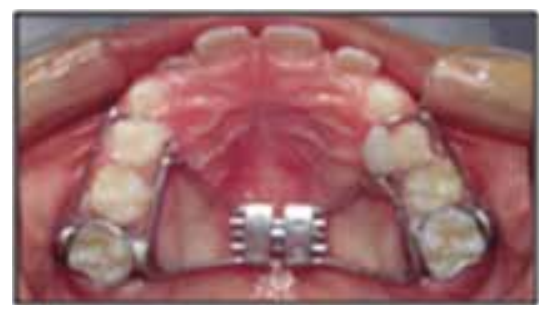

Fig. 10. Disyuntor Hyrax que nos servirá de anclaje para la tracción reversa de la maxila.

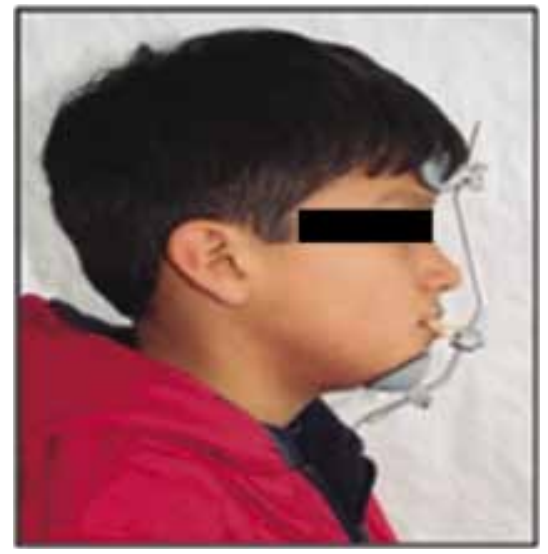

Fig. 11. Máscara facial de Petit instalada.

\section{Progresión del tratamiento}

El tratamiento empezó con la colocación de bandas en primeras molares permanentes y confección de aparato de expansión palatina tipo hyrax modificado con dos ganchos entre caninos y primeras molares deciduas en ambos lados; soldado de bandas con expansor en zona palatina, soldado de alambre 0.8 entre bandas y ganchos en zona vestibular (como anclaje). Se decidió no realizar ningún tipo de expansión rápida debido a que en el paciente ya se había realizado una expansión rápida años atrás, además que no presentaba recidiva en sentido transversal. Adaptación de máscara facial de Petit para realizar protracción maxilar. Uso de elásticos de tracción de $1 / 2$ pulgada (dos elásticos por gancho) generando una fuerza de $450 \mathrm{~g}$ por lado. El niño fue instruido en la colocación de los elásticos, rol en el cual se le notó muy cómodo y hábil. Se le encomendó a la madre supervisar el uso de la máscara facial por lo menos 16 horas al día, evitando que el niño use la máscara en horario escolar para evitar problemas de autoestima. Después de un período de 14 días se observó corrección de mordida cruzada anterior y mordida abierta bilateral en sector posterior (debido a rotación mandibular). A los 21 días después se observó mejoras en acople dentario anterior y posterior, se observó también lesión eritematosa descamativa en mentón debido al constante uso de la aparatología. Una vez cada dos semanas durante 4 meses se realizó controles evidenciándose mejoras en el resalte anterior, engranaje e intercuspidación dentaria. Se prescribió el uso de la máscara facial por 2 meses más, al cabo de dicho tiempo se realizó la evaluación del caso.

\section{Resultados obtenidos}

Se cambió el perfil de cóncavo a recto con lo que se mejoró la apariencia facial. Se corrigió la mordida cruzada anterior (Fig 11-13). En el estudio cefalométrico de reevaluación (Tabla1) encontramos que los cambios obtenidos son básicamente dentoalveolares a nivel de los incisivos superiores e inferiores, cabe recalcar que nosotros esperamos un cambio ortopédico programando un tiempo de tratamiento en esta fase de 10 meses aproximadamente, de los cuales tenemos 6 meses de tratamiento, es importante resaltar que el paciente tuvo un proceso bronquial que limitó el uso de la aparatología durante un mes, pero es un paciente muy colaborador y receptivo el cual está volviendo a retomar el tratamiento. Los cambios dentoalveolares clínicamente marcan un cambio importante dentro del esquema de tratamiento que hasta ahora recibe el paciente (Fig. 12 y 13). La estabilidad y el pronóstico son reservados debido al tipo de maloclusión presente. Las condiciones para la corrección final de la maloclusión se han visto grandemente favorecidas.

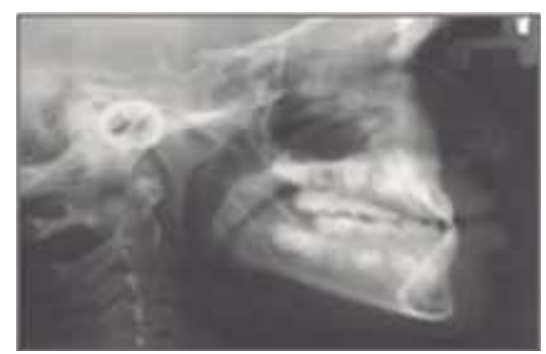

Fig. 12. Radiografía cefalométrica antes del uso de la máscara facial de Petit.

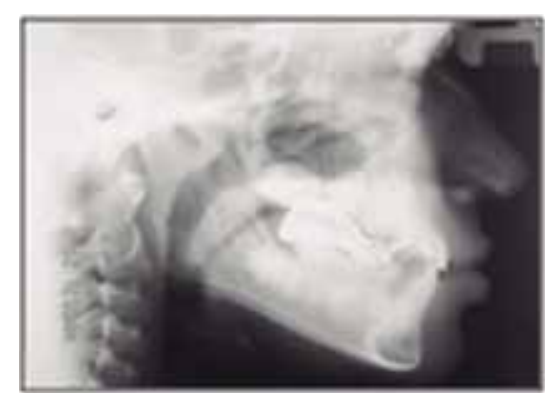

Fig. 13. Radiografía cefalométrica después del tratamiento. Nótese la corrección de la mordida cruzada anterior.

\section{Discusión}

El manejo temprano de las alteraciones dentofaciales tiene múltiples beneficios relacionados con el crecimiento y desarrollo del individuo. ${ }^{8-11}$ Quienes no favorecen la fase inicial apoyan sus ideas basados en la poca estabilidad a largo plazo de este tipo de tratamiento. ${ }^{12}$

La modificación de los patrones faciales a pesar de contar con un factor genético predominante, se hace posible gracias a una intervención meticulosa y pronta de cada caso. ${ }^{13}$ Sin embargo, es importante tener un seguimiento individualizado, tomando cada decisión basándose en la evidencia científica disponible. ${ }^{14}$

La corrección temprana de la oclusión en pacientes con maloclusión clase III puede contribuir a un cambio en el patrón neuromuscular que guiará el crecimiento restante. Se sabe que el resultado de un tratamiento es producto de la interacción de determinantes de crecimiento genéticos y cambios producidos directamente a nivel morfológico e indirectamente a través de cambios en el patrón neuromuscular. Los resultados del tratamiento inicial con máscara facial, podrían atribuirse principalmente a un desplazamiento anterior de la maxila y una reducción en el prognatismo mandibular, lo que supone un aumento en la inclinación del plano mandibular.

En el período de pos tratamiento, los cambios esqueletales tienden a disminuir y son sustituidos por la compensación dentoalveolar. El efecto del tratamiento sobre el prognatismo mandibular podría deberse a los efectos secundarios relacionados a la rotación posterior de la mandíbula, pero también al resultado de un desplazamiento temporal del cóndilo en la fosa. En ese caso, se podría explicar la rápida recaída a nivel esqueletal que se observa en algunos pacientes. El aumento de la relación sagital de la mandíbula se da por la combinación de desplazamiento hacia adelante del maxilar y una rotación posterior de la mandíbula. En algunos casos, el crecimiento del complejo maxilar inducido por la máscara facial continúa durante el proceso de retención. ${ }^{4,15}$

Tahmina muestra que la rotación de la mandíbula hacia arriba y hacia adelante, en combinación con el crecimiento y desplazamiento, está altamente asociado con resultados insatisfactorios después del crecimiento puberal en pacientes clase III en crecimiento. ${ }^{16}$

Da Silva y Pacheco sugieren que la terapia de máscara facial no normaliza el crecimiento, los pacientes reanudaron 
su patrón de crecimiento característico de clase III, caracterizado por una deficiencia maxilar. Se recomienda la sobrecorrección del resalte y de la relación molar como una herramienta para la estabilidad a largo plazo. Esta se recomienda porque luego del tratamiento estos pacientes crecen de manera similar a los no tratados con clase III, con la sobrecorrección del tratamiento se muestra buena estabilidad. ${ }^{5,17}$

En el presente caso nosotros, por experiencia propia, observamos recidiva de la mordida cruzada anterior iniciada en el recambio dentario anterior, la cual requirió de una sobrecorreción. ${ }^{2}$

\section{Conclusiones}

Siendo la mordida cruzada una maloclusión que altera el crecimiento de los maxilares, justifica en gran medida su corrección una vez identificada, requiriendo evaluación constante en corto, mediano y largo plazo, pudiendo encontrar recidiva durante el crecimiento del paciente, y este control debe extenderse hasta el cese del crecimiento, momento en el cual será necesario reevaluar el caso. Teniendo en cuenta que la terapia con máscara facial se torna incómoda para el paciente, por el largo periodo de tiempo de tratamiento, es vital la cooperación del paciente y sobretodo de los padres, los cuales juegan un rol importante en el tratamiento de este tipo de maloclusiones.

\section{Referencias bibliográficas}

1. Mahony D. Tratamiento interceptivo de maloclusiones de clase III. Ortodoncia Clínica 2001; 4(1):10-15.

2. Soldevilla LC. Corrección de mordida cruzada anterior y posterior bilateral en dentición decidua con aparatología removible adhesiva Reporte de un caso. Odontología San Marquina 2004; 8(2):49-53.

3. Escriván de Saturno L. Ortodoncia en Dentición Mixta. 1. ${ }^{a}$ ed. Caracas: Editorial Amolca; 2007.

4. Benedetti M, Bosch C, Melsen B. Changes ocurring during and after treatment of class III malocclusion with rapid palatal expansión and Face mask. World Journal of Othodontics 2002;3(3):199-212.

5. Da Silva L. Tratamiento de la maloclusión de clase III con máscara facial. Acta Odontol. Venez. 2006; 44(3):424-430.

6. Shanker S. Cephalometric A point changes during and after maxillary protraction and expansion. Am J Orthod Dentofacial Orthop. 1996; 110(4):423-430.

7. Macdonald KE. Cephalometric changes after the correction of class III malocclusion with maxillary expansion/facemask therapy. Am J Orthod Dentofacial Orthop. 1999; 116(1):13-24.

8. Brodie AG. Facial patterns a theme on variation. Angle Orthod 1946; 46(3): 75-87.
9. Ngan P, Hägg U, Yiu O, Merwin D, Wei SHY. Soft tissue and dentoskeletal profile changes associated with maxillary expansion and protraction headgear treatment. Am J Orthod Dentofacial Orthop 1996; 109: 38-49.

10. Tollaro I, Baccetti T, Franchi L. Mandibular skeletal changes induced by early functional treatment of Class III malocclusion: a superimposition study. Am J Dentofacial Orthop. 1995; 108: 525-532.

11. Deguchi T, Kuroda T, Minoshisma Y, Graber TM. Craneofacial features of patients with Class III abnormalities: growth-related changes and effects of short-term and longterm chincup therapy. Am J Orthod Dentofacial Orthop. 200; 117: 669680.

12. Chef MT. Soft and hard tissue changes alter bimaxillary surgery in chinese Class III patients. Angle Orthod 2005; 75: 959-963.

13. Vaughn GA, Mason B, Moon HB, Turley PK. The effects of maxillary protraction therapy with or without rapid palatal expansion: a prospective, randomized clinical trial. $\mathrm{Am}$ J Orthod Dentofacial Orthop. 2005; 128(3):299-309.

14. Quintero Y. Relación esquelética clase III con factor genético predominante. Reporte de un caso. Rev CES Odontología 2007; 20(2): 43-50.

15. Mew JRC. Factors influencing mandibular growth. Angle Orthod 1986; 56(1): 31-48. 